FORMATION Formation emploi

Revue française de sciences sociales

113 | janvier-mars 2011

La flexicurité à l'aune de l'approche par les capacités

\title{
Introduction : Pourquoi la flexicurité mérite-telle qu'on en débatte à partir de l'approche par les capacités?
}

Joan Miquel Verd et Josiane Vero

\section{OpenEdition}

Journals

Édition électronique

URL : http://journals.openedition.org/formationemploi/3213

DOI : 10.4000/formationemploi.3213

ISSN : 2107-0946

Éditeur

La Documentation française

Édition imprimée

Date de publication : 1 mars 2011

Pagination : 5-14

ISSN : 0759-6340

Référence électronique

Joan Miquel Verd et Josiane Vero, « Introduction : Pourquoi la flexicurité mérite-telle qu'on en débatte à partir de l'approche par les capacités ? », Formation emploi [En ligne], 113 | janvier-mars 2011, mis en ligne le 05 mai 2011, consulté le 30 octobre 2020. URL : http://journals.openedition.org/

formationemploi/3213 ; DOI : https://doi.org/10.4000/formationemploi.3213 


\section{DOSSIER}

\section{Introduction : Pourquoi la flexicurité mérite-elle qu'on en débatte à partir de l'approche par les capacités?}

par Joan Miquel Verd et Josiane Vero*

Il y a tout juste quatre ans, en 2007, Formation Emploi publiait un dossier intitulé «Pour une approche par les capacités », témoignant de l'intérêt que suscite la réflexion développée par Amartya Sen, prix Nobel d'économie en 1998, sur la justice sociale. En introduction du dossier, Robert Salais soulignait combien les idées de Sen débordaient aujourd'hui les domaines de la pauvreté et du sous-développement, où elles ont trouvé leurs premières expressions, pour entrer dans ceux de l'emploi, de la formation, de la politique sociale et du développement économique des pays riches. La même année, la flexicurité devenait un mot d'ordre Européen, dans une communication intitulée «Vers des principes communs de flexicurité : des emplois plus nombreux et de meilleure qualité en combinant flexibilité et sécurité » (Commission européenne, 2007). La commission européenne y définissait quatre composantes de politiques fructueuses en matière de flexicurité en vue de réformer les marchés du travail. La formation tout au long de la vie y était présentée comme une de ces quatre idées force.

De nombreuses réformes du marché du travail, de la formation, de la législation sociale, du droit du travail, de la négociation collective, des systèmes de
* Joan-Miquel Verd est diplômé en sciences politiques et sociologie, et en sciences économiques et de gestion. Professeur titulaire au département de sociologie de l'Université Autonome de Barcelone, il est rattaché au Centre d'études sociologique sur la vie quotidienne et le travail (QUIT). Dans le cadre du projet européen Capright, il a coordonné, avec Josiane Vero, I'atelier de travail dédié à l'analyse des parcours individuels. Sur le plan méthodologique, ses recherches portent sur l'analyse biographique et l'étude des réseaux sociaux. Sur le plan théorique, il s'interesse à la relation formation-emploi, les compétences et le lien entre protection sociale et emploi. Publications récentes: J. M. Verd, J. Vero, y M. López (2009), "Trayectorias laborales y enfoque de las capacidades. Elementos para una evaluación longitudinal de las políticas de protección social", en Sociología del Trabajo, 67, pp. 127-150 ; A. Kuznik, J. M. Verd (2010) "Investigating Real Work Situations in Translation Agencies. Work Content and Its Components", en Hermes. Journal of Language and Communication Studies, 44, pp. 25-44.

Josiane Vero est économiste, chargée d'études au Céreq. Ses travaux portent sur les politiques et les ....... 
pratiques de formation continue en entreprise, les politiques du marché du travail, les théories de la justice économique et sociale. Dans le cadre du projet européen Capright, elle a coordonné, avec Joan Miquel Verd, l'atelier de travail dédié à l'analyse des parcours individuels. Elle a récemment publié : "Trayectorias laborales y enfoque de las capacidades. Elementos para una evaluación longitudinal de las políticas de protección social", Sociología del Trabajo, 2009, $n^{\circ}$ 167, pp. 127-150, lavec Joan Miquel Verd et Martí López Andreu », et «Formation et développement professionnel, la responsabilité des entreprises en question", Actes de la conférence finale du projet Capright, Nantes, 2 et 3 décembre. http://www. capright.eu, lavec Marion Lambert, D. Subramanian et B. Zimmermann).

protection sociale sont, depuis lors, expérimentées selon des principes variés, dans les pays européens et ailleurs. Ils entendent articuler, voire compenser les politiques de flexibilité, par l'adoption de nouveaux droits et dispositifs à même d'assurer aux citoyens européens la possibilité de maîtriser leur avenir professionnel. Comment analyser les transformations portées par la stratégie de flexicurité tout en se démarquant des rhétoriques qu'il est possible de repérer dans les communications et rapports officiels ? Quels sont concrètement les déplacements qui s'opèrent en son nom et quelles sont leurs incidences sur les destins professionnels ? Comment s'articule travail, formation, emploi et sécurité à l'échelle du parcours des personnes ? Comment rendre compte du rôle de la formation dans la sécurisation des parcours professionnels? À partir de quels indicateurs? Quelles seraient les étapes à franchir pour que les politiques de formation, d'emploi et de protection sociale favorisent le développement d'un environnement permettant aux individus d'exercer des choix au cours de leur carrière ? Enfin, que nous apprennent, de ce point de vue, les expériences étrangères?

Répondre à ces questions a constitué l'un des enjeux d'une recherche collective et interdisciplinaire développée sous la conduite de Robert Salais. Elle vise à proposer une autre voie, à relancer l'Europe sociale sur de nouvelles bases en développant l'approche par les capacités (AC) introduite par A. Sen. C'est pourquoi ce dossier s'appuie, pour l'essentiel, et c'est l'une de ses originalités, sur les travaux conduits à partir du programme européen dit « Capright $»^{1}$.

Dans un premier temps, nous avançons quelques arguments pour montrer en quoi l'AC peut constituer une théorie sociale pertinente pour penser alternativement les réformes de l'État social placées sous le signe de la flexicurité. La deuxième partie présentera les principaux thèmes abordés par les contributions rassemblées dans ce numéro.

\section{L'APPROCHE PAR LES CAPACITÉS}

Trouver la meilleure façon d'articuler flexibilité et sécurité est aujourd'hui envisagé comme un enjeu politique de premier rang. Pour assurer la cohérence et la pérennité des réformes, il est indispensable de trouver un juste équilibre entre flexibilité et sécurité. Avec Salais (2006, p. 432), nous défendons la thèse suivante : pour parvenir à un tel équilibre, «l'agenda social de l'Europe doit être basé sur une théorie sociale qui lui soit propre. Il doit exister un rapport évident entre cette théorie et la politique qui s'en déduit selon des implications qui soient tout aussi claires ». Avec lui, nous défendons l'idée que l'AC peut constituer cette théorie. Sans chercher à réduire l'éventail des contributions de cette approche, il est néanmoins possible de dégager quelques raisons qui justifient le recours à la perspective des capacités pour analyser le paradigme de la flexicurité. On soulignera d'abord un point de méthode, puis un point qui tient aux finalités de l'approche par les capacités.

L'approche par les capacités a, d'abord, le mérite d'offrir une vision intégrée et générale de la question sociale qui permet de lier l'éthique à l'économie. Elle offre un cadre pour la conception, la mise en œuvre, mais aussi l'évaluation de l'action publique. En ce sens, elle constitue un front épistémologique contre les positions qui se disent simplement descriptives, mais

${ }^{1}$ Capright (2007-2010) est un projet européen de recherche relevant du $6^{\mathrm{e}}$ Programme Cadre de la DG Recherche de l'Union européenne. Coordonné par Robert Salais, il vise à relancer l'Europe sociale sur de nouvelles bases en appliquant l'approche par les capacités, introduite par A. Sen, dans les domaines de l'emploi, du travail, de la formation et de la protection sociale : Resources, rights and capabilities: in search of social foundations for Europe-CIT4-CT-2006-028549 
qui sont toujours susceptibles de manipulation idéologique. Car, à y regarder de plus près, ce qui compte et ce qui doit entrer dans la convention d'évaluation quand il s'agit de penser les arrangements sociaux ou juger des situations individuelles est tributaire d'un jugement de valeur, que celui-ci soit revendiqué ou passé sous silence. C'est un premier point à souligner.

$\mathrm{Au}$ centre de cette théorie évaluative, il y a la notion centrale de capacité. Elle désigne l'éventail des libertés réellement accessibles à une personne en vue de conduire la vie qu'elle entend mener. Ce concept ne saurait être séparé des trois dimensions qui le composent, «l'une fondée sur la liberté, l'autre articulée à l'épanouissement humain et une troisième liée à la réalisation des droits » (de Munck, 2008, p. 23), parce que les individus doivent avoir la possibilité réelle - au-delà des droits formels - de vivre conformément à l'idée qu'ils se font d'une bonne vie. Dès lors, la référence, et l'aiguillon de la justice dans l'approche par les capacités, se trouve dans l'étendue des libertés réelles dont chaque personne dispose en vue de choisir la vie qu'elle entend mener.

Le deuxième point qui doit être signalé est qu'elle est, comme le rappelle de Munck, "savamment inachevée », ce qui la rend particulièrement féconde en vue d'une réappropriation dans le débat européen (De Munck, 2008, p. 29). Au-delà des orientations générales que l'approche par les capacités trace pour l'évaluation de l'action publique, le «cadre proposé est concret, au sens où il s'attache à toutes les conditions institutionnelles, matérielles et personnelles qui, dans la situation observée, influent les capacités » (Salais, 2005, p. 15). Cette approche doit, pour fonctionner comme un cadre évaluatif, être contextualisée. Rechercher les conditions favorables au développement économique et humain qui soient à la fois efficaces et justes ne découle pas «de la définition substantielle qu'en pourrait donner un théoricien en fauteuil ni même un expert dans une salle de réunion de l'ONU. Il faut plonger le concept de capacité en situation, dans un contexte particulier, pour lui donner son opérativité » (De Munck, ibid.). Les politiques de flexicurité promues par l'Union européenne peinent à intégrer les exigences du terrain et les besoins des personnes. Au contraire, l'AC met l'accent sur la liberté réelle des participants au processus démocratique. L'incomplétude de la propo- sition de Sen se présente comme la condition même d'actualisation de sa vision constructive de la démocratie (Bonvin, 2005).

L'approche par les capacités offre une alternative à la vision de la flexicurité telle qu'elle est promue à l'échelon européen. Une lecture des orientations de celle-ci semble révéler qu'elle est encadrée par un double consensus, à la fois sur le point de départ (une insuffisance des taux d'emploi pour atteindre l'objectif de compétitivité fixé au sommet de Lisbonne), et le point d'arrivée (une adaptation nécessaire des travailleurs aux exigences du marché du travail). La conception est instrumentale, comme le souligne Salais (2005, p. 12) : "On ne saurait mieux dire que l'homme doit être mis au service du marché. » On a peine, en effet, à croire que remettre à marche forcée, une personne dans un emploi de courte durée, mal payé et sans qualification, va accroître l'étendue des possibilités de vie et de travail de celui ou celle à qui on l'a imposé. Cette vision est en contradiction avec la promotion des capacités des personnes et de leurs libertés (Salais et Supiot, 2003 ; Salais 2005 ; Supiot 2010). Dans l'approche par les capacités, l'objectif de justice sociale subordonne nécessairement l'organisation économique aux exigences de la vie en société. La réappropriation de ce débat, sous le prisme de l'approche par les capacités, abandonne le primat du marché envers et contre tout. Le message de Sen est clair, le seul aiguillon éthiquement légitime par rapport auquel l'action publique doit-être élaborée, mise en œuvre et évaluée, cette seule référence, c'est l'étendue des libertés réelles dont dispose une personne pour choisir et mener la vie qu'elle entend mener. Il ne s'agit pas là de libertés formelles, mais de libertés réelles d'action, c'est-à-dire de libertés positives. L'action publique est ainsi appelée à développer les opportunités à disposition des salariés tout en ne cherchant pas à leur imposer des manières d'être ou de vivre spécifiques. Pour cela, l'AC met l'accent sur deux dimensions essentielles de la liberté réelle (1) le développement des opportunités (empowerment) grâce auxquelles les personnes acquièrent les moyens de la liberté de choix (2) le respect de la liberté processuelle, grâce à laquelle elles restent maîtres de leur choix (Bonvin et Farvaque, 2008). Au cœur de l'AC émerge une conception particulièrement exigeante de 
la démocratie qui se démarque nettement de la vision de la flexicurité (Bonvin, 2005).

Ce déplacement du curseur peut sembler modeste, mais il implique des changements importants sur la manière de concevoir et d'évaluer les situations des personnes. Insister sur les libertés réelles c'est, d'une part, aller au-delà d'une conception fondée sur les ressources ou les droits formels dont disposent les personnes. Ainsi, le Droit Individuel à la Formation (DIF) est une ressource importante, mais il ne suffit pas à assurer la liberté réelle des personnes. Toutes les personnes n'ont pas les mêmes aptitudes à convertir des droits formels en liberté réelle de choix. Parmi les facteurs qui impactent l'exercice de cette liberté réelle interviennent, au premier rang, les politiques conduites par les entreprises. À travers les opportunités de formation qu'elles offrent et les dispositifs de délibération qu'elles aménagent, les entreprises jouent comme un facteur de conversion des ressources en capacités (Lambert et al., 2010 ; Lambert et al., 2011). Ainsi, les capacités des personnes dépendent autant de la possession de ressources que de la présence de facteurs de conversion favorables. Ces derniers peuvent être liés à des caractéristiques environnementales, sociales ou individuelles.

Insister sur les libertés réelles c'est, d'autre part, distinguer la manière dont les personnes se comportent effectivement (fonctionnements ou accomplissements) et ce qu'elles sont réellement libres de faire (capacités). Un même fonctionnement peut résulter de la présence ou de l'absence de libertés réelles. Par exemple, quelqu'un qui expérimente un changement d'horaires de travail, sans avoir d'autres opportunités que d'accepter cette décision prise de manière unilatérale par l'employeur, ne jouit pas d'une liberté réelle de travailler. En revanche, un salarié qui disposera d'un éventail de possibilités alternatives dont il aura pu délibérer avec son employeur jouira, quant à lui, d'une plus grande liberté réelle, bien qu'il puisse travailler sur les mêmes horaires. Ainsi l'approche de Sen repose sur la distinction entre droits formels (ressources), droits réels (capacités) et comportements effectifs (fonctionnements).

Il importe donc d'accroître les ressources, mais aussi de renforcer le pouvoir des individus de choisir, de mener la vie à laquelle ils aspirent. L'attention aux personnes, à leurs droits, à la formation et à l'expression de leurs capacités prend une importance essentielle dès lors que leur place dans le système productif est beaucoup moins prédéterminée et définitive qu'à l'époque fordiste, que les liens entre l'entreprise et le salarié deviennent plus volatiles. Les itinéraires professionnels se différencient rapidement en fonction des réussites ou des échecs de ce nomadisme ; le partage du subi et du volontaire est variable et inégalitaire. Dès lors, la référence et l'aiguillon de la justice dans l'approche par les capacités se trouvent bien dans l'étendue des libertés réelles dont chaque personne dispose en vue de choisir la vie qu'elle entend mener.

Parler de libertés réelles des personnes, au moment où l'Europe, comme le monde entier, est plongée dans une crise financière et économique d'ampleur inégalée depuis la crise de 1929 pourrait sembler totalement futile. Au contraire, la thèse défendue par Sen est que : «Les libertés réelles sont non seulement les fins premières du développement, mais aussi un de ses principaux moyens. » [Sen, 2000, p. 10] Comme Salais l'a souligné, "faire en sorte que chacun, tel qu'il est et là où il est, puisse réaliser ses possibilités est une condition même de la poursuite du développement économique » (Salais, 2005, p. 12). Sen pense cette dynamique en termes de libertés réelles.

C'est donc à l'aune des libertés réelles que les travaux proposés dans ce dossier se fondent, et qu'ils invitent à évaluer les politiques de flexicurité.

\section{PRÉSENTATION DES ARTICLES : LES DÉFIS DE LA FLEXICURITÉ SELON L'APPROCHE PAR LES CAPACITÉS}

Pour les auteurs de ce dossier, l'approche par les capacités constitue une perspective sérieuse pour relancer l'Europe sociale sur de nouvelles bases ; de même, elle constitue une proposition décisive à la manière de concevoir et d'évaluer l'articulation entre flexicurité et sécurité. Pour d'autres auteurs, elle ne serait qu'une chimère, un avatar de la conception rawlsienne ou un effet de mode qui ne serait pas en mesure de résister durablement à l'épreuve du temps (Gamel, 2007). Mais qu'on incline à la défendre ou à la pourfendre, 
l'intérêt d'y réfléchir est facile à identifier à quiconque s'efforce de repenser en profondeur les fonctions de l'État social face à la crise à laquelle celui-ci est confronté, à quiconque cherche à reconfigurer le couplage entre flexibilité et sécurité de manière à améliorer les situations réelles des personnes, à quiconque cherche à offrir une alternative novatrice aux orientations actuelles promues dans le cadre de la stratégie européenne pour l'emploi. L'objectif de ce numéro est de contribuer à ce que cette proposition puisse faire l'objet d'un débat serein et informé.

Si tel est bien le projet social et politique qui soustend les recherches présentées dans ce numéro dédié à la flexicurité, celle-ci n'est pas évoquée par Sen lui-même dans ses écrits. Ce qui est proposé dans ce numéro ne relève donc pas d'une simple application des travaux de Sen, mais d'une ré-appropriation et ré-interprétation de ses réflexions transposées dans un domaine de l'action publique européenne. Il s'agit donc d'un objet à construire dans une démarche de critique scientifique de l'action publique. Il est clair, d'autre part, que les innovations radicales qu' implique une opérationnalisation de l'approche par les capacités représentent un défi de poids. Sen n'a jamais proposé de méthode clé en main, de boîte à outils universelle pour évaluer les capacités, laissant au chercheur le soin de définir une méthodologie adaptée pour saisir empiriquement la liberté réelle d'action des personnes face à leur diversité, aux spécificités sociales, historiquement construites et géographiquement enracinées. Dans ce contexte, les obstacles à surmonter et les questions à approfondir ne manquent pas. C'est dire l'ampleur des défis à surmonter. C'est à eux que s'attaquent les contributions rassemblées dans ce numéro. Elles visent à évaluer les réformes du marché du travail et de la protection sociale et à offrir une alternative qui fasse des libertés réelles une valeur cardinale tout en accordant à la question des institutions une importance décisive.

À l'appui de cette réflexion, Jean-Michel Bonvin, Éric Moachon et Josiane Vero ouvrent le débat sur les fondements normatifs de la politique commune de flexicurité. Ils analysent les deux seuls indicateurs dynamiques de flexicurité que sont la sécurité de l'emploi et le progrès des qualifications. Ils s'intéressent, au travers de ce que l'on veut mesurer, au type de flexicurité que la Commission cherche à promouvoir à l'échelle européenne. La lecture des orientations de la flexicurité, à travers le prisme de ces deux indicateurs, semble révéler que celle-ci est encadrée par un double consensus, à la fois sur le point de départ (une insuffisance des taux d'emploi pour atteindre l'objectif de compétitivité fixée au sommet de Lisbonne) et le point d'arrivée (une adaptation nécessaire des travailleurs aux exigences du marché du travail). La conception de la formation, telle qu'elle y est développée, revêt un caractère instrumental : elle attend de la personne tout ce qui peut servir les objectifs d'adaptabilité, de polyvalence et de responsabilité. Dans le cadre de cet article, les auteurs éprouvent la solidité d'une telle orientation à partir de l'approche par les capacités. Ils discutent les limites de ces fondements normatifs qui privilégient flexibilité plutôt que liberté réelle des travailleurs, employabilité plutôt que capacité, et capital humain plutôt que développement professionnel. Ils proposent une conception alternative de la sécurité de l'emploi et du rôle qu'est susceptible d'y jouer la formation et concluent en présentant quelques repères afin d'élaborer des indicateurs davantage inspirés par l'approche par les capacités.

Pascal Caillaud et Bénédicte Zimmermann soulignent, à l'appui d'une double analyse juridique et sociologique de la situation française, toute l'importance de la question de l'articulation entre flexibilité et sécurité, dans l'activité même de travail, pour développer la liberté d'entreprendre mais encore la liberté de travailler et de se développer professionnellement. Ils notent, à cet égard, que l'attention au travail ne semble pas avoir influencé les orientations de la stratégie de flexicurité. Alors que celle-ci se concentre sur les dimensions juridiques et institutionnelles (l'emploi, la formation, les politiques du marché du travail), les auteurs déportent le débat vers le lien entre flexicurité et travail. Autrement dit, alors que les composantes de la flexicurité placent au second rang la liberté de travailler et de se développer professionnellement, c'est précisément le souci de mieux prendre en compte cette dimension qui pousse les auteurs à s'intéresser au couplage flexibilité / sécurité dans le travail. Ce mouvement d'une attention à l'emploi à une attention au travail implique de se centrer sur les cadres institutionnels, les parcours biographiques mais aussi sur la politique, notamment de formation, mise en œuvre dans les entreprises et ses conséquences pour les 
salariés. La démarche met en évidence le rôle décisif de l'entreprise et montre que si la formation intervient comme un élément de sécurisation des parcours, elle ne constitue qu'un élément parmi d'autres. Elle exige aussi la capacité à s'exprimer des intéressés, mais aussi d'autres capacités telle que la capacité à réaliser un travail de qualité dans un emploi de qualité, et la capacité à équilibrer vie familiale et vie professionnelle. Il devient dès lors essentiel, pour les auteurs, d'envisager la formation sous l'angle du développement professionnel. C'est une des grandes forces de cette contribution que de questionner directement la responsabilité des entreprises en matière de formation, du point de vue de la sécurité et du développement professionnel des salariés. Il reste que, par rapport à la voie dans laquelle la flexicurité est engagée, cette recherche, centrée sur le travail et le développement professionnel, incite à un réagencement des cadres de réflexion en matière de flexicurité et à la mise en œuvre d'autres principes d'observation et d'action qui permettraient de mieux comprendre le rôle de la formation et la façon dont elle s'inscrit dans des organisations et des contextes institutionnels différenciés.

Les trois articles suivants, privilégiant une approche quantitative, s'intéressent plus particulièrement au lien entre la formation et la sécurisation des parcours sur le marché du travail en Wallonie, Catalogne et en Argentine.

L'article de Bernard Conter et Jean-François Orianne explore, en Wallonie, une double hypothèse : d'une part, le rôle de la formation dans la sécurisation des parcours telle qu'elle est thématisée dans le discours sur la flexicurité ; de l'autre, les effets de la formation dans l'extension de la liberté de choix promue par l'A.C. Alors que la première engage à évaluer la rapidité des transitions et l'efficacité des dispositifs en termes de sortie du chômage, la seconde conduit à apprécier les possibilités de choix des individus. Le développement des dispositifs de formation destinés aux chômeurs n'aboutit pas aux mêmes conclusions selon la perspective adoptée. Si les stagiaires de la formation professionnelle semblent plus nombreux à accéder à l'emploi que les demandeurs d'emploi qui n'ont pas bénéficié de tels programmes, la formation semble étendre de manière assez marginale l'espace des possibles en termes d'accès à l'emploi. Mais plus que la formation, c'est l'expérience professionnelle antérieure qui constitue le principal vecteur de retour à l'emploi.

Marti Lopez-Andreu et Joan Miquel Verd analysent, pour leur part, en Catalogne, l'impact de la formation non formelle et celui de la formation formelle sur les changements de situation sur le marché du travail (emploi ou chômage), les changements de contrat de travail ou de catégorie professionnelle. Leur contribution recoupe, à certains égards, les conclusions de Bernard Conter et Jean-François Orianne. Tout comme en Wallonie, l'effet de la formation apparaît moins décisif que celui du parcours sur le marché du travail. Les profondes réformes allant dans le sens de la flexibilité, introduites en Espagne depuis 1985, ont généré plusieurs profils de travailleurs présentant des parcours très différenciés À l'issue d'une typologie, distinguant la variété des parcours observés sur le marché du travail Catalan, la formation est analysée au regard des effets qu'elle produit dans l'emploi et dans l'aménagement de certaines transitions. Les résultats de ces travaux montrent que les caractéristiques sociales de ces parcours semblent annuler, dans certains cas, les effets positifs potentiels de la formation suivie.

La flexibilité ne fait pas débat qu'en Europe. En Argentine, aussi, comme nous le rappelle Corina Enriquez Rodriguez, qui s'attache à analyser le modèle argentin confronté à une flexibilité accrue. Caractérisé par un important secteur informel, le marché du travail argentin est marqué par une faible disponibilité des programmes d'activation de l'emploi et d'assurancechômage. Mais là aussi, en analysant les parcours sur le marché du travail, elle montre le faible impact des programmes de formation. Les résultats de ses travaux font écho aux résultats que signalent les contributions de Marti Lopez-Andreu et Joan Miquel Verd d'une part, et de Bernard Conter et Jean-François Orianne, d'autre part. Les politiques publiques comme la mise en place de programmes de formation se révèlent inopérantes pour développer les qualifications et la capacité pour l'emploi des profils de personnes aux parcours les plus précaires.

Les trois articles précédents, en se fondant sur des données qui n'ont pas été directement collectées pour évaluer les capacités, concluent dans chaque cas sur des résultats modestes de la formation sur l'emploi et 
l'aménagement des transitions sur le marché du travail et les possibilités de choix qui y sont associées.

Ces résultats, qui convergent pour attester du faible lien entre la formation et l'emploi, n'en invitent pas moins à s'interroger sur la pertinence des dispositifs d'observation aveugles à l'environnement de la formation et au rôle qu'est susceptible de jouer les formes d'organisation du travail et de gestion des ressources humaines dans la sécurisation des parcours. Car comme le montrent les travaux de Caillaud et Zimmermann, «La sécurité des salariés ne se joue pas seulement dans l'emploi et l'aménagement de transitions sur le marché du travail ; elle se forge aussi dans le travail à proprement parler et les possibilités de développement professionnel qu'offre ce dernier $»$. Dans ce cadre, la formation comme facteur de sécurisation ne prend sens que dans la construction d'un parcours, dans une projection dans l'avenir rendue possible par des formes d'organisation du travail et de gestion des carrières qui replacent l'acquisition de savoirs dans un processus plus global de développement professionnel.

Les résultats des recherches conduites dans ce numéro appellent plus ou moins explicitement des changements profonds dans les dispositifs d'observation susceptibles d'analyser le rôle de la formation dans la sécurisation des parcours. La première conséquence est que l'investigation empirique de la flexicurité à l'aune des capacités devrait se centrer sur l'analyse des parcours indissociable de données longitudinales. Or, dans la batterie d'indicateurs de flexicurité, seuls deux indicateurs revêtent une dimension dynamique (Bonvin et al., 2009). La deuxième conséquence est le souci de mieux prendre en compte la liberté dans la sphère de l'emploi mais aussi dans celle du travail en arrimant cette dernière à sa dimension organisationnelle (Zimmermann, 2011). La troisième conséquence est que le type d'information sur lequel se baser pour analyser le rôle de la formation dans la sécurisation des parcours demanderait à dépasser une observation centrée sur un seul versant du marché du travail, celui de la demande d'emploi, observée à partir des comportements individuels. La sécurisation des parcours professionnels dans une perspective de développement de la liberté réelle ne se limite pas qu'à une question d'employabilité individuelle. Intégrer d'autres niveaux d'analyse, et notamment l'autre versant tout aussi important, celui de l'offre d'emploi par les employeurs et la gestion de la main-d'œuvre que les entreprises développent, apparaît décisif (Salais, 2010). Tous deux sont d'ailleurs traversés par des réformes qui ne prennent sens que dans des contextes institutionnels donnés. En d'autres termes, croiser ces trois dimensions permet de dessiner les axes structurants de l'investigation empirique de la sécurisation des parcours à l'aune de l'approche par les capacités.

Les auteurs de ce dossier ne prétendent pas à la neutralité. L'approche par les capacités montre d'ailleurs que la neutralité n'est pas possible, comme évoqué plus haut. Le formatage de l'information, et le système de catégories qui représente la situation d'une personne du point de vue de l'action publique à entreprendre sont décisifs (Salais, 2010). Si ces auteurs estiment important de contribuer à nourrir la réflexion sur une approche alternative de la flexicurité fondée sur l'AC, c'est parce qu'ils sont convaincus qu'elle désigne une direction dans laquelle l'articulation entre flexibilité et sécurité doit évoluer.

Mais ce numéro spécial n'est pas conçu comme un plaidoyer. En donnant un aperçu limpide et documenté d'un débat d'actualité, en exhibant les points de repère normatifs à partir desquels les politiques à l'œuvre sont évaluées, et en discutant leur conformité à des principes de justice donnés, il veut fournir au lecteur une base pour former ses propres convictions et ouvrir quelques voies susceptibles de redonner sens à l'objectif de justice sociale sur lequel devrait se baser une réforme des politiques de flexicurité. Pareille ambition oblige non seulement à rompre avec «les idéologies qui réduisent l'homme à du matériel ou du capital humain » (Supiot, 2010, p. 23), mais à inverser la perspective pour «indexer l'économie sur les besoins de l'homme» (Ibid, p. 25) et renouer, comme Alain Supiot (2010) nous y invite, avec l'esprit de la Déclaration de Philadelphie de 1944. 
Bibliographie et pour en savoir plus...

Abbateccola E., Lefesne F., Verd J.M. and Vero J. (éds) (2012), «Individual Working Lives and the Capability Approach. An overview across Europe », Transfer, Vol. 18 (1), à paraître.

Bakhshi P., Brouillet A-S, Duray-Soundron C., Dubois J.-L. (2008) (éds), Repenser l'action collective : une approche par les capabilités, (Réseau Impact, coll. «Éthique économique »), Paris, L'Harmattan, 280 p.

Bartelheimer P., Büttner R., Moncel N. (éds) (2009), Sen-sitising life course research?, Exploring Amartya Sen's capability concept in comparative research on individual working lives, Actes du Séminaire CAPRIGHT, Goettingen, 24-25 Septembre 2008, Net-doc, avril, $\mathrm{n}^{\circ} 50$.

Bénicourt E. (2004), "Contre Amartya Sen », L’économie Politique, $\mathrm{n}^{\circ} 23$, juillet, pp. 72-84.

Bénicourt E. (2005), « Sen : du texte à ses interprétations », L'économie Politique, ${ }^{\circ} 27$, juillet, pp.52-59.

Bevort A., Lallement M., Nicole-Drancourt C. (éds) (2006), «Flexicurité. La protection de l'emploi en débat », Problèmes politiques et sociaux, $\mathrm{n}^{\circ} 931$, décembre.

Bonvin J.-M. (2005), « La démocratie dans l'approche d'Amartya Sen ", L'Économie politique, vol. 3, $\mathrm{n}^{\circ} 27$, pp. 24-37.

Bonvin J.-M. (2005), «Sortir de l'homooeconomicus: la voie anthropologique d'Amartya Sen », Finance \& Bien Commun, n² 22, pp. 73-79.

Bonvin J.-M. et Farvaque N. (2008), Amartya Sen. Une politique de la liberté, Paris, Michalon, 128 p.

Bonvin J.-M (2009), « Analyser les formes contemporaines de négociation. La contribution de l'approche par les capacités », Négociations Vol. 2, n 12 , pp. 245-255.

Bonvin J.-M. et Orton M. (éds) (2009), « Activation policies and organisational innovation in the capability perspective ", International Journal of Sociology and Social Policy, December, vol. 29, issue 11-12.

Bonvin J.-M. et Veille P. (2009), «Une flexicurité au service des capacités des citoyens européens », La Revue de l'Ires, Vol. 63, n 4, pp. 17-33.

Corteel D., Lambert M., Vero J., Zimmermann B. (2009), « Capability for learning in French companies », in Bartelheimer P., Büttner R., Moncel N. éds (2009), Sen-sitising life course research?, Exploring Amartya Sen's capability concept in comparative research on individual working lives, Actes du Séminaire CAPRIGHT, Goettingen, 24-25 Septembre 2008, Net-doc, avril, $\mathrm{n}^{\circ} 50$.

Commission européenne (2007), Vers des principes communs de flexicurité : des emplois plus nombreux et de meilleure qualité en combinant flexibilité et sécurité, Bruxelles, [COM(2007) 359 final].

De Munck J., Zimmermann B. (2008) (éds), La liberté au prisme des capacités. Amartya Sen au-delà $d u$ libéralisme, Paris, Éditions de l'EHESS, coll. « Raisons Pratiques ».

De Munck J. (2008), « Qu'est-ce qu'une capacité ? » in De Munck, J. et Zimmermann, B. (éds.), La liberté au prisme des capacités. Amartya Sen au-delà du libéralisme, Paris, Éditions de l'EHESS, coll. « Raisons pratiques », pp. 21-50.

Dupriez V., Orianne J.-F., Verhoeven M. (éds) (2008), De l'école au marché du travail, l'égalité des chances en question, Berne : Peter Lang, 411 p.

Farvaque N. et Robeyns I. (2005), «L'approche alternative d'Amartya Sen. Réponse à Emmanuelle Bénicourt », L'économie Politique, $\mathrm{n}^{\circ} 27$, juillet, pp. 38-51.

Farvaque N. (2005), Action Publique et approche par les capacités : une analyse des dispositifs et trajectoires d'insertion, thèse de sciences économiques, université de Marne la Vallée. 
Formation Emploi (2007), numéro Spécial «Pour une approche par les capacités », $\mathrm{n}^{\circ} 98$, avril-juin.

Gamel C. (2007), «Que faire de "l'approche par les capacités" ? Pour une lecture "rawlsienne" de l'approche de Sen», Formation Emploi, n ${ }^{\circ}$ 98, pp. 141-150.

Gazier B. (2010), «Adieu "flexicurité" ? La sécurisation des parcours professionnels » in P. (ed), Ressources humaines pour sortie de crise, Presses de Sciences Po, pp. 205-222.

La Revue de l'IRES (2009), numéro spécial, «Flexicurité, sécurisation des parcours professionnels et protection sociale », Vol. $63, \mathrm{n}^{\circ} 4$.

Lambert M., Vero J. (2010), «Aspirer à se former, la responsabilité des entreprises en question », BrefCéreq, $\mathrm{n}^{\circ} 279$, novembre.

Lambert M., Vero J., Zimmermann B. (2011), «Formation et développement professionnel, la responsabilité des entreprises en question ", Actes de la conférence finale Capright, http://www.capright.eu/

L'Économie politique (2005), «Faut-il lire Amartya Sen ? », $\mathrm{n}^{\circ} 27$, juillet.

Maggi Germain, N. (2009), « La capacité du salarié à occuper un emploi », Droit Social, ${ }^{\circ} 12$, décembre, pp. 1-12.

Monnet E. (2007), "La théorie des "capabilités" d'Amartya Sen face au problème du relativisme ", Tracés. Revue de Sciences humaines, ${ }^{\circ} 12$, Faut-il avoir peur du relativisme?, mai, pp. 103-120.

Perez C. et Vero J. (2006), «L'accès à la formation en entreprise au regard des modes de gestion de la main-d'œuvre », Travail et Emploi, juillet-septembre, $\mathrm{n}^{\circ} 107$, pp. 59-71.

Salais R. (2010), «La donnée n'est pas un donné pour une analyse critique de l'évaluation chiffrée de la performance », Revue française d'administration publique, $\mathrm{n}^{\circ} 135$, pp. 497-515.
Salais R. (2007), « À la recherche de principes novateurs pour l'action publique », Formation Emploi, $\mathrm{n}^{\circ} 98$, pp. 5-8.

Salais R. (2008), «Capacités, base informationnelle et démocratie délibérative. Le (contre) exemple de l'action publique européenne, in De Munck J., Zimmermann B. (éds), La liberté au prisme des capacités. Amartya Sen au-delà du libéralisme.

Salais R. (2005), «Le projet européen à l'aune des travaux de Sen », L'économie Politique, $\mathrm{n}^{\circ} 27$, juillet, pp. $8-23$.

Salais R. et Villeneuve R. (éds) (2006), Développer les capacités des hommes et des territoires en Europe, Éditions de l'Anact, Lyon, 456 p.

Salais R. (2003), "Work and Welfare: Toward a Capability-based Approach" in Zeitlin J. and Trubek D. (éds.), Governing Work and Welfare in a New Economy : European and American Experiments, Oxford, Oxford University Press, pp. 317-344.

Salais R. et Supiot A. (2003), "L'unione non fa il lavoro", Il Manifesto, p. 12.

Sen A. K. (1992), Repenser l'inégalité, Seuil, 282 p.

Sen A. K. (1999), Éthique et économie, PUF, 364 p.

Sen A. (2000), Un nouveau modèle économique. Développement, justice, liberté, Paris, Odile Jacob poches, $479 \mathrm{p}$.

Sen A. (2003), L'économie est une science morale, La Découverte, $126 \mathrm{p}$.

Sen A. (2005), Rationalité et liberté en économie, Odile Jacob, 559 p.

Sen A. (2010), L'idée de Justice, Flammarion, collection « Essais ».

Sigot J.-C. et Vero J. (2009), « Démocratiser la formation continue dans l'entreprise : le rôle de l'information, des entretiens professionnels et des supports collectifs », Bref-Céreq, n 260. 
Subramanian D., Verd J.M., Vero J. et Zimmermann B. (éds) (2012) "Management policies, work and capabilities", International Journal of Manpower, à paraître.

Supiot A. (éd.) (1999), Au-delà de l'emploi. Transformations du travail et devenir du droit $d u$ travail en Europe, Paris, Flammarion, 321 p.

Supiot A. (2010), L'esprit de Philadelphie. La justice sociale face au marché total, Éditions du Seuil.

Verd J.M., Vero J., López Andreu M. (2009), "Trayectorias laborales y enfoque de las capacidades. Elementos para una evaluación longitudinal de las políticas de protección social”, Sociología del Trabajo, $\mathrm{n}^{\circ}$ 167, pp. 127-150.

Vero J. (2002), Mesurer la pauvreté à partir des Concepts de biens premiers, de réalisations primaires et de capabilités de base. Le rôle de l'espace d'information dans l'identification de la pauvreté des jeunes en phase d'insertion professionnelle, thèse en économie mathématique et économétrie, EHESS-GREQAM.

Vielle P. (2007), «Flexicurité : redéfinir la sécurité des citoyens européens », Revue belge de sécurité sociale, $\mathrm{n}^{\circ} 4$, pp. $735-775$.

Vladimirova K. (éd.) (2010), Transformations in Labour: Social Security and Social Dialogue, Capright Conference proceedings, Sofia, 23-24 March 2009 Ciela.

Zimmermann B. (2006), « Pragmatism and the capability approach. Challenges in social theory and empirical research », European Journal of Social Theory, 9 (4), pp. 467-484.

Zimmermann (2010), Ce que Travailler veut dire: une sociologie des capacités et des parcours professionnels, Economica, 233 p. 